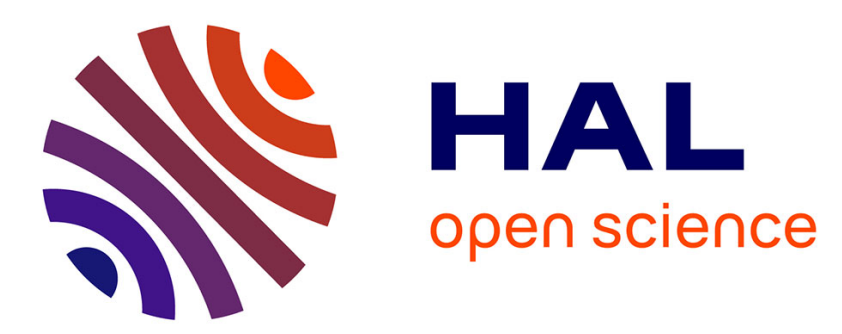

\title{
Design of broadband time-domain impedance boundary conditions using the oscillatory-diffusive representation of acoustical models
}

\author{
Florian Monteghetti, Denis Matignon, Estelle Piot, Lucas Pascal
}

\section{To cite this version:}

Florian Monteghetti, Denis Matignon, Estelle Piot, Lucas Pascal. Design of broadband time-domain impedance boundary conditions using the oscillatory-diffusive representation of acoustical models. Journal of the Acoustical Society of America, 2016, 140 (3), pp.1663 - 1674. 10.1121/1.4962277 . hal-01530759

\author{
HAL Id: hal-01530759 \\ https://hal.science/hal-01530759
}

Submitted on 31 May 2017

HAL is a multi-disciplinary open access archive for the deposit and dissemination of scientific research documents, whether they are published or not. The documents may come from teaching and research institutions in France or abroad, or from public or private research centers.
L'archive ouverte pluridisciplinaire HAL, est destinée au dépôt et à la diffusion de documents scientifiques de niveau recherche, publiés ou non, émanant des établissements d'enseignement et de recherche français ou étrangers, des laboratoires publics ou privés. 


\title{
Design of broadband time-domain impedance boundary conditions using the oscillatory-diffusive representation of acoustical models
}

\author{
Florian MONTEGHETTI ${ }^{1}$ \\ ONERA - The French Aerospace Lab, 31055 Toulouse, France \\ Denis MATIGNON \\ ISAE-SUPAERO, Université de Toulouse, 31055 Toulouse, France \\ Estelle PIOT and Lucas PASCAL \\ ONERA - The French Aerospace Lab, 31055 Toulouse, France
}

\begin{abstract}
A methodology to design broadband time-domain impedance boundary conditions (TDIBCs) from the analysis of acoustical models is presented. The derived TDIBCs are recast exclusively as first-order differential equations, well-suited for high-order numerical simulations. Broadband approximations are yielded from an elementary linear least squares optimization that is, for most models, independent of the absorbing material geometry. This methodology relies on a mathematical technique referred to as the oscillatory-diffusive (or poles and cuts) representation, and is applied to a wide range of acoustical models, drawn from duct acoustics and outdoor sound propagation, which covers perforates, semiinfinite ground layers as well as cavities filled with a porous medium. It is shown that each of these impedance models leads to a different TDIBC. Comparison with existing numerical models, such as multi-pole or extended Helmholtz resonator, provides insights into their suitability. Additionally, the broadly-applicable fractional polynomial impedance models are analyzed using fractional calculus.

PACS numbers: 43.28.Py, 43.28.En, 43.28.Js.
\end{abstract}

Copyright notice. This is an author-prepared document.

Copyright 2016 Acoustical Society of America. This article may be downloaded for personal use only. Any other use requires prior permission of the author and the Acoustical Society of America.

The following article appeared in "The Journal of the Acoustical Society of America 140, 1663 (2016)" and may be found at http://dx.doi.org/10.1121/1.4962277.

\section{INTRODUCTION}

The modeling of sound absorption in the time domain (as opposed to the frequency domain) through a timedomain (acoustic) impedance boundary condition (TDIBC) has several attractive features for computational aeroacoustics, ${ }^{1}$ such as the ability to handle broadband noise ${ }^{2}$ in one fell swoop, or to be coupled with nonlinear partial differential equations (e.g. Euler). Nonetheless, in practice, theoretical $^{3,4}$ and empirical ${ }^{5,6}$ models are hardly ever known in closed form in the time domain, and, when they are, the corresponding convolutions are numerically costly to compute. TDIBCs, whose development can be traced back to the early 90s, are an attempt to deal with these two difficulties, by providing simple yet broadly applicable numerical impedance models, as well as efficient numerical methods to tackle convolutions. They have found use in several areas, such as duct acoustics, ${ }^{7}$ outdoor sound propagation, ${ }^{8}$ and room acoustics. ${ }^{9}$

The earliest numerical impedance models were comprised of a single polynomial or rational fraction, which yields in the time domain an ordinary differential equation (ODE) between the acoustic pressure and normal velocity. A second-degree polynomial was used by Davis ${ }^{10}$ to model an open pipe. Tam and Auriault ${ }^{11}$ broadened his study by considering a three-parameter model (proportionalintegral-derivative). Inspired by the progress made in the computational electromagnetics community, Özyörük et $a l .{ }^{12}$ followed a heuristic approach to propose an admissible rational fraction of degree 4 ; as it can match the behavior of a ceramic tubular liner until the second resonance, it has been hailed as the first "broadband" model.

The need for a generic, efficient, broadband TDIBC has led to the introduction of a new family of models, known as "multi-pole". They consist of a discrete sum of elementary first or second-order low-pass systems. The number $N$ of systems, as well as their respective gains and poles, are degrees of freedom (DoF) of the TDIBC, which translates as a considerable versatility. Moreover, admissibility conditions are straightforwardly verified, which is not the case for rational fractions expressed with polynomials (especially of higher degree). The drawback is that they lead to $N$ elementary convolutions. Borrowed from the computational electromagnetic community, ${ }^{13}$ so-called "recursive" (recurrent) convolution techniques have been employed by many authors. ${ }^{14,15,16,8,17}$ Bin et al. ${ }^{18}$ used an alternative implementation, relying instead on $N$ additional differential equations. A comprehensive study by Dragna et al. ${ }^{19}$ showed the benefit of this technique, known as the auxiliary differential

\footnotetext{
${ }^{1}$ Electronic mail: florian.monteghetti@onera.fr
} 
equations method, over recursive convolution.

However, multi-pole models are purely numerical. An alternative was proposed by Rienstra, ${ }^{20}$ who introduced, on physical grounds, the extended Helmholtz resonator (EHR) model. Apart from its polynomial part, it consists in the impedance of a cavity filled with a non-dispersive medium having a frequency-independent dissipation. Its similarity to a single DoF liner model eases the fitting process. Several implementations have been proposed. ${ }^{21,7,22}$

Most of the available TDIBCs are designed to be generic: this implies that they must be tuned based on available experimental data, which often proves delicate, as impedance measurements tend to be associated with significant uncertainties. ${ }^{23}$

The objective of this paper is to present how a time-domain analysis of physical models (known to yield satisfactory fits in the frequency domain) can be used to design a time-local, parsimonious, tailored, broadband TDIBC. The analysis relies on a technique known as the diffusive, or poles and cuts, ${ }^{24}$ representation, referred to herein as the oscillatorydiffusive representation. It is demonstrated on a wide range of models, which covers perforated plates, cavities, as well as porous media. The link with existing TDIBCs is investigated.

This paper is organized as follows. Sec. II recalls some basics of impedance modeling. Section III lists the physical impedance models covered, and demonstrates the wide applicability of so-called fractional polynomial models. These are covered in Sec. IV, which lays out the derivation of the TDIBC using the diffusive representation. Sec. V reuses the same principles to cover more advanced perforation and semi-infinite ground layer models. Lastly, Sec. VI covers cavity models through the oscillatory-diffusive representation, and highlights their specificity.

\section{GENERALITIES ON IMPEDANCE MODELING}

Absorbing materials, approximated as locally reacting, are commonly characterized in the frequency domain by defining a surface acoustic impedance $\hat{z}^{s}$ that links the acoustic pressure $p$ and normal inward velocity $u_{n}$ through

$$
\forall s \in \mathbb{C}: \Re[s]>\zeta, \hat{p}(s)=\hat{z}^{s}(s) \hat{u}_{n}(s) .
$$

The $e^{+s t}\left(e^{+\mathrm{j} \omega t}\right)$ convention is used at synthesis for the Laplace (Fourier) transform; the abscissa of convergence $\zeta$ is 0 for all the models covered herein. Provided that the causality condition is met, (1) translates in the time domain as the causal convolution

$$
\forall t>0, p(t)=\left(z^{s} \star u_{n}\right)(t),
$$

which is a linear integro-differential equation between $p$ and $u_{n}$. The material is then modeled as a linear timeinvariant (LTI) system, of input $u_{n}$, output $p$ and causal impulse response $z^{s}$. The scope of this paper is restricted to this linear framework, the focus being on recasting (2) as a more convenient set of ODEs.

Through empirical models, (2) may be used to account for the presence of a basic flow, as long as it is viscous; for instance, Kirby and Cummings ${ }^{25}$ proposed a model which formally reduces to $\hat{z}^{s}\left(s, u_{*}\right)$, where $u_{*}$ is the basic flow friction velocity. However, a departure from LTI modeling is invariably caused by: an inviscid basic flow, modeled with the Ingard-Myers boundary condition; ${ }^{26}$ nonlinear phenomena, such as vortex shedding, that arise from high sound pressure levels. ${ }^{27}$ An example of nonlinear impedance model is that of Melling, ${ }^{5}$ in which the impedance exhibits a dependency on the acoustic velocity itself: $\hat{z}^{s}\left(s, u_{n}\right)$.

Admissibility criteria must be met for (2) to describe a passive absorbing material, ${ }^{28,20}$ namely reality, passivity, causality, and stability. No attempt is made herein to rigorously account for these criteria, which are verified by all the models covered; two mere remarks are given. Firstly, the Laplace transform is the framework of choice for avoiding mathematical technicalities as it is always a holomorphic function $^{29}$ (within the right half-plane), while the Fourier transform may actually be a tempered distribution, which is trickier to manipulate. Secondly, every model that uses a viscous correction proportional to $\sqrt{\omega}$ (instead of $\sqrt{\mathrm{j} \omega}$ ), originally introduced by Ingard, ${ }^{5}$ fails the reality condition due to a lack of hermitian symmetry, i.e. $\hat{z}^{s}\left(s^{*}\right) \neq \hat{z}^{s}(s)^{*}$, where ${ }^{*}$ denotes the complex conjugate. Examples include the Maa model (Eq. 4 of Ref. 30), and Eqs. 9,13,17,22 of Ref. 31. Throughout this paper, for every multi-valued function, the principal branch (i.e. that which coincides with the real-valued function over the real axis) is used.

\section{ACOUSTIC IMPEDANCE MODELS}

The purpose of this section is to describe the acoustic impedance models $\hat{z}^{s}$ covered in this paper. The configuration considered herein is a perforated plate, backed by cavities of length $l_{c}$ and diameter $d_{c}$. Its surface acoustic impedance can be split as, assuming the perforation flow is incompressible, ${ }^{32}$

$$
\hat{z}^{s}=\frac{\hat{z}^{p}}{\sigma}+\hat{z}^{b},
$$

where $\sigma$ is the porosity, $\hat{z}^{p}$ is the perforation impedance (also known as a partition impedance), and $\hat{z}^{b}$ is the impedance of the backing cavity (filled with air or a porous medium), expressed as

$$
\hat{z}^{b}(s)=\mathrm{j} \hat{z}_{c}(s) \cot \left[-k_{c}(s) l_{c}\right]=\hat{z}_{c}(s) \operatorname{coth}\left[\mathrm{j} k_{c}(s) l_{c}\right],
$$

where $k_{c}$ is the propagation wavenumber within the cavity, and $\hat{z}_{c}$ is the characteristic impedance of the cavityfilling medium. In outdoor sound propagation, $\hat{z}^{p}=0$, and a common approximation is the semi-infinite ground layer, whereby wave reflection is neglected, which leads to

$$
\hat{z}^{s}(s)=\hat{z}_{c}(s) .
$$


Models for $\hat{z}^{p}$ and $\left(\hat{z}_{c}, k_{c}\right)$ are given in Sec. A and B, respectively. Their high and low-frequency approximations are systematically given, as these are specifically covered in Sec. IV.

\section{A. Perforation impedance models}

The impedance of a perforation of length $l$ and diameter $d$ has originally been derived by Crandall, ${ }^{3}$ who considered a Stokes flow within an infinite circular cylinder:

$$
\begin{aligned}
\hat{z}^{p}(s) & =\rho_{0} l s\left[1-\Phi\left(k_{v} d / 2\right)\right]^{-1} \\
& =3 \frac{\rho_{0} l v}{(d / 2)^{2}}+2 \frac{\rho_{0} l \sqrt{v}}{d / 2} \sqrt{s}+\rho_{0} l s+\mathscr{O}\left[\frac{1}{\left|k_{v} d / 2\right|}\right] \\
& =8 \frac{\rho_{0} l v}{(d / 2)^{2}}+\frac{4}{3} \rho_{0} l s+\mathscr{O}\left[\left|k_{v} d / 2\right|^{4}\right],
\end{aligned}
$$

where $\Phi(x):=\frac{2}{x} \frac{I_{1}}{I_{0}}(x)\left(I_{n}\right.$ is the modified Bessel function of the first kind of order $n), k_{v}:=\sqrt{s / v}$ is the wavenumber associated to viscous diffusion, $\rho_{0}$ the basic flow density, and $v$ the kinematic viscosity. The dimensionless quantity $\left|k_{v} d / 2\right|$ is the Stokes number, and is proportional to the ratio of the diameter $d$ to the acoustic boundary layer thickness. When the perforation is instead a slit of width $b$, the model is formally similar (chap. 4 of Ref. 32):

$$
\begin{aligned}
\hat{z}^{p}(s) & =\rho_{0} l s\left[1-\Psi\left(k_{v} b / 2\right)\right]^{-1} \\
& =\frac{\rho_{0} l v}{(b / 2)^{2}}+\frac{\rho_{0} l \sqrt{v}}{b / 2} \sqrt{s}+\rho_{0} l s+\mathscr{O}\left[\frac{1}{\left|k_{v} b / 2\right|}\right] \\
& =3 \frac{\rho_{0} l v}{(b / 2)^{2}}+\frac{6}{5} \rho_{0} l s+\mathscr{O}\left[\left|k_{v} b / 2\right|^{4}\right]
\end{aligned}
$$

where $\Psi(x):=\tanh (x) / x$. These two models, (6) and (9), serve as a basis to cover more complex materials. ${ }^{33}$

In 2007, Atalla and Sgard ${ }^{31}$ proposed to model perforates in the same fashion as rigid-frame porous media:

$$
\hat{z}^{p}(s)=\rho_{0} l s \alpha(s),
$$

where $\alpha$ is the dynamic tortuosity, for which they suggested the model of Johnson et al. (chap. 5 of Ref. 32):

$$
\alpha(s)=\alpha_{\infty}+\frac{\sigma \phi}{\rho_{0}} \frac{1}{s} \sqrt{1+s \tau},
$$

where $\phi$ denotes the flow resistivity, $\alpha_{\infty}$ the tortuosity, and $\tau:=v\left[\frac{\rho_{0} \alpha_{\infty}}{\sigma \phi \Lambda / 2}\right]^{2}$ a characteristic time expressed with the viscous characteristic length $\Lambda$, taken as $d / 2$ or $b / 2$ for perforated plates. The model (12) is shown able to recover many existing models (such as the Beranek Ingard model), provided that $\alpha_{\infty}$ is modified accordingly. Its high and lowfrequency approximations read

$$
\begin{aligned}
& \hat{z}^{p}(s) \underset{+\infty}{=} \sigma \phi l \sqrt{\tau} \sqrt{s}+\alpha_{\infty} \rho_{0} l s+\mathscr{O}[1 / \sqrt{|s \tau|}] \\
& =\phi \sigma l+\alpha_{\infty} \rho_{0} l s\left[1+\frac{\sigma \phi \tau}{2 \alpha_{\infty} \rho_{0}}\right]+\mathscr{O}\left[|s \tau|^{2}\right] \text {. }
\end{aligned}
$$

Adjustment for radiation, viscosity and interaction effects is commonly achieved by correcting the length $l .^{5,31}$

\section{B. Backing medium impedance models}

Miki ${ }^{6}$ proposed an empirical model applicable to many fibrous porous material; to enforce the reality condition, it must be written as

$$
\begin{aligned}
& \frac{\hat{z}_{c}}{z_{0}}(s)=1+\frac{0.070}{\cos \frac{b \pi}{2}}\left[\frac{2 \pi \phi}{s}\right]^{b} \quad(b=0.632) \\
& \mathrm{j} k_{c}(s)=\frac{s}{c_{0}}\left[1+\frac{0.109}{\cos r \frac{\pi}{2}}\left(\frac{2 \pi \phi}{s}\right)^{r}\right](r=0.618),
\end{aligned}
$$

where $z_{0}:=\rho_{0} c_{0}$ is the characteristic impedance of air.

In their recent thorough study of impedance models for outdoor sound propagation, Dragna and Blanc-Benon ${ }^{28}$ showed that five porous medium models can be written as (see table I of Ref. 28):

$$
\begin{aligned}
& \frac{\hat{z}_{c}}{z_{0}}(s)=q_{z}\left[\frac{\left(s+\omega_{1}\right)\left(s+\omega_{2}\right)}{s\left(s+\omega_{3}\right)}\right]^{1 / 2} \\
& \underset{+\infty}{=} q_{z}\left[1+\frac{\omega_{1}+\omega_{2}-\omega_{3}}{2} \frac{1}{s}\right]+\mathscr{O}\left[\frac{1}{|s|^{2}}\right] \\
& =q_{z} \sqrt{\frac{\omega_{1} \omega_{2}}{\omega_{3}}} \frac{1}{\sqrt{s}}+\mathscr{O}[\sqrt{|s|}] \\
& \mathrm{j} k_{c}(s)=q_{k} \frac{s}{c_{0}}\left[\frac{\left(s+\omega_{1}\right)\left(s+\omega_{3}\right)}{s\left(s+\omega_{2}\right)}\right]^{1 / 2} \\
& \underset{+\infty}{=} q_{k} \frac{s}{c_{0}}\left[1+\frac{\omega_{1}+\omega_{3}-\omega_{2}}{2} \frac{1}{s}\right]+\mathscr{O}\left[\frac{1}{|s|}\right] \\
& =q_{k} \frac{s}{c_{0}}\left[\sqrt{\frac{\omega_{1} \omega_{3}}{\omega_{2}}} \frac{1}{\sqrt{s}}\right]+\mathscr{O}\left[|s|^{3 / 2}\right],
\end{aligned}
$$

where $q_{z}$ and $q_{k}$ are two non-dimensional positive coefficients and the angular frequencies $\omega_{i}$ are positive.

A common model for semi-infinite ground layers is the socalled variable porosity model: ${ }^{28}$

$$
\frac{\hat{z}_{c}}{z_{0}}(s)=\sqrt{\omega_{0}} \frac{1}{\sqrt{s}}+\omega_{1} \frac{1}{s},
$$

where the angular frequencies $\omega_{i}$ are positive.

By considering a Stokes flow with thermal effects, Bruneau derived a wavenumber for an air-filled isothermal cylindrical cavity of diameter $d_{c}$ (see Sec. 3.7 of Ref. 4):

$$
\begin{aligned}
\mathrm{j} k_{c}(s) & =\frac{s}{c_{0}}\left[\frac{1+(\gamma-1) \Phi\left(k_{\kappa} d_{c} / 2\right)}{1-\Phi\left(k_{v} d_{c} / 2\right)}\right]^{1 / 2} \\
& =\frac{s}{c_{0}}\left[1+\frac{\sqrt{v}}{d_{c} / 2}\left(\frac{\gamma-1}{\sqrt{\operatorname{Pr}}}+1\right) \frac{1}{\sqrt{s}}\right]+\mathscr{O}[1] \\
& =\frac{s}{c_{0}}\left[\frac{2 \sqrt{2} \sqrt{\gamma \nu}}{d_{c} / 2} \frac{1}{\sqrt{s}}\right]+\mathscr{O}\left[\left|k_{v} d / 2\right|^{3}\right]
\end{aligned}
$$


where $\gamma$ denotes the ratio of specific heat, and $\kappa$ the thermal diffusivity. For an adiabatic cavity, thermal effects can be neglected: the Prandtl number $\operatorname{Pr}$ is infinite, $\phi\left(k_{\kappa} d_{c} / 2\right)$ is null. Additionally, neglecting viscous effects leads to the inviscid wavenumber

$$
\mathrm{j} k_{c}(s)=\frac{s}{c_{0}} .
$$

\section{FRACTIONAL POLYNOMIAL IMPEDANCE MODELS IN THE TIME DOMAIN}

Sec. III has shown that most of the high and lowfrequency approximations of both perforation and semiinfinite ground layer models are so-called fractional polynomial impedance models (FPIMs), defined as

$$
(\Re[s]>0) \quad \hat{z}_{1}^{s}(s)=a_{-\alpha} \frac{1}{s^{\alpha}}+a_{0}+a_{\beta} s^{\beta}+a_{1} s,
$$

where the coefficients $a$. are non-negative, and $(\alpha, \beta)$ is in ] $0,1\left[^{2}\right.$. (Note that describing (29) as a fractional polynomial is an abuse of terminology when $\alpha \neq 0$.) This section focuses on the design of a TDIBC for FPIMs, which are a first step toward more complex models. It is voluntarily detailed so as to enable a clearer presentation of the more general developments presented in Sec. V and VI.

The FPIM (29) exhibits two fractional terms, namely $s^{-\alpha}$ and $s^{\beta}$. In each of the physical models reviewed in Sec. III, at the notable exception of the empirical Miki model (16), $\alpha$ or $\beta$ equals $1 / 2$. Physically, a fractional term $\sqrt{s}$ is the manifestation of a diffusion process, be it viscous or thermal. For instance, in perforations or cavities, this diffusion process occurs within a (thermal) viscous boundary layer, whose thickness $\delta_{v}\left(\delta_{\kappa}\right)$ is given by (see Sec. 2.5.2 of Ref. 4) $\delta_{\bullet}=\sqrt{2} /|\sqrt{s / \bullet}|$, where $\bullet$ denotes $v(\kappa)$. As a rule, the main contribution of this fractional term is on the resistance (see, for instance, the Crandall model (7)).

This section is broken down in four parts. Sec. A covers the admissibility of (29). The design of the TDIBC for $s^{-\alpha}$ is described in Sec. B, while $s^{\beta}$ is covered in Sec. C. Optimization strategies are finally discussed in Sec. D. Sec. A is rather technical, and can be omitted at first reading, as it is not necessary to understand the subsequent sections.

\section{A. Admissibility in the time domain}

The purpose of this section is to justify the admissibility of (29), which has recently been challenged in the literature. ${ }^{20}$ As $z_{1}^{s}$ obeys the admissibility conditions mentioned in Sec. II, it is a causal and tempered distribution. However, a second, more constructive argument, draws from a theory of fractional calculus. ${ }^{34}$ The starting point is to rewrite (29) as

$$
\hat{z}_{1}^{s}(s)=a_{-\alpha} \hat{h}_{1}^{\alpha}(s)+a_{0}+a_{\beta} \hat{h}_{1}^{1-\beta}(s) s+a_{1} s,
$$

where the function $\hat{h}_{1}^{\alpha}$ is defined in the frequency domain as

$$
(\Re[s]>0) \quad \hat{h}_{1}^{\alpha}(s):=\frac{1}{s^{\alpha}},
$$

and in the time domain as the causal function

$$
(t>0) \quad h_{1}^{\alpha}(t):=\frac{1}{\Gamma(\alpha) t^{1-\alpha}}
$$

with $\Gamma$ denoting the Euler gamma function. The fractional integration is defined using the Riemann-Liouville formula (valid for any real number $\alpha \geq 0$ ):

$$
\mathrm{I}^{\alpha}\left[u_{n}\right](t):=\left(h_{1}^{\alpha} \star u_{n}\right)(t)=\int_{0}^{t} h_{1}^{\alpha}(t) u_{n}(t-\tau) \mathrm{d} \tau
$$

However, the definition of the fractional derivative requires more caution. A mere generalization of (33) for $\alpha<0$, known as the Riemann-Liouville fractional derivative, is unpractical (i.e. is only properly defined in the space of causal distribution). ${ }^{34}$ Including a non-null initial condition on $u_{n}$ leads to the fractional derivative in the sense of $\mathrm{Ca}$ puto, ${ }^{35,34}$ defined for $\left.\beta \in\right] 0,1[$ as

$$
\mathrm{d}^{\beta}\left[u_{n}\right]:=\left(h_{1}^{1-\beta} \star \dot{u}_{n}\right)=\mathrm{I}^{1-\beta}\left[\dot{u}_{n}\right],
$$

where Newton's notation is used for the time derivative of $u_{n}$. The Caputo derivative (34) consists in applying the Riemann-Liouville formula to the time derivative $\dot{u}_{n}$ of $u_{n}$.

Both (33) and (34) are real-valued, causal and passive operators; in the time domain, (30) converts into the following fractional differential equation:

$$
p=a_{-\alpha} \mathrm{I}^{\alpha}\left[u_{n}\right]+a_{0} u_{n}+a_{\beta} \mathrm{d}^{\beta}\left[u_{n}\right]+a_{1} \dot{u}_{n} .
$$

However insightful (35) may be, its numerical integration is cumbersome as is. The next two sections, Secs. B and C, focus on the design of an efficient TDIBC for the fractional integral and derivative, using the diffusive representation.

\section{B. Fractional integral in the time domain}

In this section, we design a TDIBC for the fractional integral in (29), denoted

$$
(\Re[s]>0) \quad \hat{z}_{1}^{-\alpha}(s):=a_{-\alpha} s^{-\alpha}=a_{-\alpha} \hat{h}_{1}^{\alpha}(s) .
$$

The diffusive representation of $h_{1}^{\alpha}$, presented in Sec. 1, enables to formulate a continuous TDIBC in Sec. 2, the discretization of which leads to the TDIBC in Sec. 3.

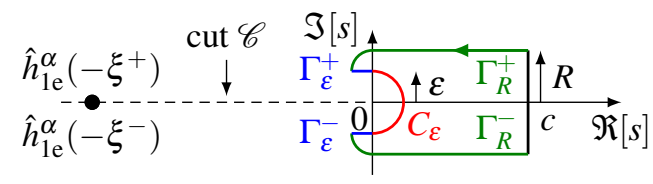

FIG. 1. (Color online) Bromwich path used to compute (37). The jump of $\hat{h}_{1 e}^{\alpha}$ across the cut is at the origin of the diffusive representation (40). 


\section{Diffusive representation}

To derive a more computationally practical representation of $\hat{h}_{1}^{\alpha}$, the starting point is the Bromwich integral

$$
(t>0) \quad h_{1}^{\alpha}(t)=\frac{1}{2 \mathrm{j} \pi} \int_{c-\mathrm{j} \infty}^{c+\mathrm{j} \infty} \hat{h}_{1}^{\alpha}(s) e^{s t} \mathrm{~d} s .
$$

The analytical computation of (37) uses a well-known technique in complex analysis: ${ }^{36}$ the conjoint use of the residue theorem with Jordan's lemma on a closed path. The subtlety (and, subsequently, the richness of the model) lies in the fact that $\hat{h}_{1}^{\alpha}$ is only defined in the right half-plane $\Re[s]>0$ : any extension to the left half-plane leads to a multivalued function. The choice of a cut $\mathscr{C}$ on $\mathbb{R}_{\text {- }}$ enables to define an extended function $\hat{h}_{1 e}^{\alpha}$, which coincides with $\hat{h}_{1}^{\alpha}$ on the right half-plane and preserves its hermitian symmetry:

$$
\left(s \in \mathbb{C} \backslash \mathbb{R}_{-}\right) \quad \hat{h}_{1 e}^{\alpha}(s):=\frac{1}{s^{\alpha}} .
$$

On the path depicted in Fig. 1, which circumvents the cut, the residue theorem gives, for all $\varepsilon>0$ and $R>\varepsilon$ :

$$
\int_{c-\mathrm{j} R}^{c+\mathrm{j} R} \hat{h}_{1 e}^{\alpha}(s) e^{s t} \mathrm{~d} s+\int_{\Gamma_{R} \cup \Gamma_{\varepsilon} \cup C_{\varepsilon}} \hat{h}_{1 e}^{\alpha}(s) e^{s t} \mathrm{~d} s=0 .
$$

After taking the limit $R \rightarrow \infty$ (Jordan's lemma) then $\varepsilon \rightarrow 0$, the only remaining contribution on $h_{1}^{\alpha}(t)$ is that of the cut, which can be merged into one integral:

$$
h_{1}^{\alpha}(t)=\int_{0}^{\infty} \mu_{1}^{\alpha}(\xi) e^{-\xi t} \mathrm{~d} \xi .
$$

The diffusive weight $\mu_{1}^{\alpha}$, which is the result of $\hat{h}_{1 e}^{\alpha}$ being multivalued, is proportional to the jump of $\hat{h}_{1 e}^{\alpha}$ across the cut $\mathscr{C}:{ }^{24}$

$$
\begin{aligned}
(\xi>0) \quad \mu_{1}^{\alpha}(\xi) & =\frac{1}{2 \mathrm{j} \pi}\left[\hat{h}_{1 e}^{\alpha}\left(-\xi^{-}\right)-\hat{h}_{1 e}^{\alpha}\left(-\xi^{+}\right)\right] \\
& =\frac{\sin (\alpha \pi)}{\pi} \times \frac{1}{\xi \alpha} .
\end{aligned}
$$

The identity (40) is known as the diffusive representation (also representation by cut) of the convolution kernel $h_{1}^{\alpha}$; in the frequency domain, computing its Laplace transform yields

$$
\hat{h}_{1}^{\alpha}(s)=\int_{0}^{\infty} \frac{\mu_{1}^{\alpha}(\xi)}{s+\xi} \mathrm{d} \xi,
$$

provided that switching the order of integration is licit, i.e. that the diffusive weight obeys the following wellposedness condition

$$
\int_{0}^{\infty} \frac{\left|\mu_{1}^{\alpha}(\xi)\right|}{1+\xi} \mathrm{d} \xi<\infty
$$

The theoretically exact representation (40) gives an insight into the dynamic of the fractional integral (33): it consists in a continuum of first-order dynamics, which extends from high frequencies ( $\xi \gg 1$, short memory) to low frequencies ( $\xi \ll 1$, long memory).

\section{Continuous TDIBC}

The diffusive representation (43) enables us to rewrite the fractional impedance model (36) as a continuum of firstorder low-pass systems

$$
\hat{z}_{1}^{-\alpha}(s)=a_{-\alpha} \int_{0}^{\infty} \frac{\mu_{1}^{\alpha}(\xi)}{s+\xi} \mathrm{d} \xi,
$$

or, equivalently, in the time domain

$$
z_{1}^{-\alpha}(t)=a_{-\alpha} \int_{0}^{\infty} \mu_{1}^{\alpha}(\xi) e^{-\xi t} \mathrm{~d} \xi,
$$

which leads to the following continuous TDIBC

$$
p(t)=a_{-\alpha} \int_{0}^{\infty} \mu_{1}^{\alpha}(\xi)\left(e^{-\xi} \star u_{n}\right)(t) \mathrm{d} \xi,
$$

where the original convolution $z_{1}^{-\alpha} \star u_{n}$ is "reduced" to an infinity of simpler convolutions, namely $e^{-\xi} \star u_{n}$. The computational interest of (47) stems from the fact that it can be recast using only first-order ODEs:

$$
p(t)=a_{-\alpha} \int_{0}^{\infty} \mu_{1}^{\alpha}(\xi) \varphi_{\xi}(t) \mathrm{d} \xi,
$$

where the diffusive (also memory) variables $\varphi_{\xi}$ are defined as

$$
\varphi_{\xi}(t):=\left(e^{-\xi} \star u_{n}\right)(t)+\varphi_{0}(\xi) e^{-\xi t},
$$

and solve the first-order ODE

$$
\dot{\varphi}_{\xi}(t)=-\xi \varphi_{\xi}(t)+u_{n}(t) \quad \varphi_{\xi}(0)=\varphi_{0}(\xi),
$$

with null initial condition $\varphi_{0}(\xi)=0$. $\left(\dot{\varphi}_{\xi}\right.$ denotes the time derivative of $\varphi_{\xi}$.) The continuous TDIBC consists of $(48,50)$. Mathematically, the fractional integration (33) has been expressed as an observer of the infinite-dimensional state space representation (50).

\section{Discrete TDIBC}

The corresponding discrete TDIBC is simply deduced from a discretization of the continuous $\operatorname{TDIBC}(45,46,48)$, which yields

$$
\begin{array}{ll}
(\Re[s]>0) & \hat{z}_{1, \text { num }}^{-\alpha}(s)=a_{-\alpha} \sum_{k=1}^{N_{\varphi}} \frac{\tilde{\mu}_{k}}{s+\xi_{k}} \\
(t>0) & z_{1, \text { num }}^{-\alpha}(t)=a_{-\alpha} \sum_{k=1}^{N_{\varphi}} \tilde{\mu}_{k} e^{-\xi_{k} t} \\
(t>0) & p(t)=a_{-\alpha} \sum_{k=1}^{N_{\varphi}} \tilde{\mu}_{k} \varphi_{k}(t),
\end{array}
$$

where the $N_{\varphi}$ poles $\xi_{k}$ and gains $\tilde{\mu}_{k}$ remain to be chosen (see Sec. D). Each of the $N_{\varphi}$ diffusive variables $\varphi_{k}:=\varphi_{\xi_{k}}$ obeys the first-order dynamic (50) with null initial condition $\varphi_{k}(0)=0$. 


\section{Fractional derivative in the time domain}

In this section, we design a TDIBC for the fractional Caputo derivative in (29), denoted

$$
(\Re[s]>0) \quad \hat{z}_{1}^{\beta}(s):=a_{\beta} s^{\beta}=a_{\beta} \hat{h}_{1}^{1-\beta}(s) s .
$$

The diffusive representation of $\hat{h}_{1}^{1-\beta}$ computed in Sec. B readily yields

$$
\hat{z}_{1}^{\beta}(s)=a_{\beta}\left[\int_{0}^{\infty} \frac{\mu_{1}^{1-\beta}(\xi)}{s+\xi} \mathrm{d} \xi\right] s .
$$

The corresponding continuous TDIBC is therefore

$$
(t>0) \quad p(t)=a_{\beta} \int_{0}^{\infty} \mu_{1}^{1-\beta}(\xi)\left(e^{-\xi} \star \dot{u}_{n}\right)(t) \mathrm{d} \xi .
$$

By contrast with (47), the elementary convolutions are $e^{-\xi} \star \dot{u}_{n}$ instead of $e^{-\xi} \star u_{n}$. However, by using the identity

$$
e^{-\xi} \star \dot{u}_{n}=-\xi\left[\frac{u_{n}(0)}{\xi} e^{-\xi}+e^{-\xi \cdot \star u_{n}}\right]+u_{n},
$$

the expression (56) can be recast as

$$
p(t)=a_{\beta} \int_{0}^{\infty} \mu_{1}^{1-\beta}(\xi)\left[-\xi \varphi_{\xi}(t)+u_{n}(t)\right] \mathrm{d} \xi,
$$

where the diffusive variables $\varphi_{\xi}$ still obey (50), but with non-null initial condition $\varphi_{0}(\xi)=u_{n}(0) / \xi$. Note that this non-null initial condition is the very difference between the fractional derivative in the sense of Caputo and that of Riemann-Liouville, used by Ostashev et al. ${ }^{16}$ and Blanc et al. ${ }^{37}$

Consequently, the discrete TDIBC reads:

$$
\begin{aligned}
& (\Re[s]>0) \quad \hat{z}_{1, \text { num }}^{\beta}(s)=a_{\beta}\left[\sum_{k=1}^{N_{\varphi}} \frac{\tilde{\mu}_{k}}{s+\xi_{k}}\right] s \\
& (t>0) \quad p(t)=a_{\beta} \sum_{k=1}^{N_{\varphi}} \tilde{\mu}_{k}\left[-\xi_{k} \varphi_{k}(t)+u_{n}(t)\right],
\end{aligned}
$$

where the $N_{\varphi}$ poles $\xi_{k}$ and gains $\tilde{\mu}_{k}$ remain to be chosen (see Sec. D).

\section{Optimization strategy}

The DoF of the TDIBCs (53) and (60) are the $N_{\varphi}$ poles $\xi_{k}$ and gains $\tilde{\mu}_{k}$. The numerical model should be a fair approximation of the theoretical one, which translates for $\hat{z}_{1, \text { num }}^{-\alpha}$ as

$$
\sum_{k=1}^{N_{\varphi}} \frac{\tilde{\mu}_{k}}{\mathrm{j} \omega+\xi_{k}} \simeq \hat{h}_{1}^{\alpha}(\mathrm{j} \omega)
$$

on a band of interest $\left[\omega_{\min }, \omega_{\max }\right]$, for $N_{\varphi}$ as small as possible. On typical aeroacoustical applications, ${ }^{2}$ an approximation can be considered broadband if the bandwidth is above $10 \mathrm{kHz}$. As the approximation problem $\hat{z}_{1, \text { num }}^{\beta}(\mathrm{j} \omega) \simeq$ $\hat{h}_{1}^{1-\beta}(\mathrm{j} \omega) \mathrm{j} \omega$ is formally identical to (61), the same optimization process applies. In particular, the same gains can be used to fit both $\hat{h}_{1}^{\alpha}$ and $\hat{h}_{1}^{-\alpha}$. Note that the discrete TDIBCs obey a convergence property, i.e. $\hat{z}_{\text {num }} \rightarrow \hat{z}$ on $\left[\omega_{\min }, \omega_{\max }\right]$ for $N_{\varphi} \rightarrow \infty$.

To compute the gains $\tilde{\mu}_{k}$, the direct use of the analytical expression (42) of $\mu_{1}^{\alpha}$ with numerical quadratures proves not practical, as it leads to non-parsimonious approximations. A simple yet efficient method consists in a least-square fit in the frequency domain: the cost function is defined as ${ }^{24}$

$$
J(\tilde{\mu}, \xi)=\int_{\omega_{\min }}^{\omega_{\max }} w(\omega)\left|\sum_{k=1}^{N_{\xi}} \frac{\tilde{\mu}_{k}}{\mathrm{j} \omega+\xi_{k}}-\hat{h}_{1}^{\alpha}(\mathrm{j} \omega)\right|^{2} \mathrm{~d} \omega,
$$

where $w$ is a weight function. In practice, to approximate $\hat{h}_{1}^{\alpha}$, it is not necessary to optimize simultaneously both the gains and the poles: the latter can be chosen. The optimization procedure then goes as follows. Firstly, a distribution of $N_{\varphi}$ poles $\left(\xi_{k}\right)_{k}$ is chosen. Typically, a linear or logarithmic placement over $\left[\omega_{\min }, \omega_{\max }\right]$ is satisfactory. Secondly, the optimal gains $\tilde{\mu}_{\text {opt }}$ are computed from a minimization of $\tilde{\mu} \mapsto J(\tilde{\mu}, \xi)$. As this is a (overdetermined) linear least squares optimization problem, it is directly solved through a pseudo-inverse, without iterations.

An illustration for $\sqrt{\mathrm{j} \omega}$ is proposed in Fig. 2, for respectively two and six poles linearly placed between $10 \mathrm{~Hz}$ and $10 \mathrm{kHz}$. In this case, only a few poles are enough to achieve a broadband approximation. Note that, since $\hat{h}_{1}^{\alpha}$ is independent of the considered absorbing material (i.e. of the coefficients $a$.), then so are the gains $\tilde{\mu}_{k}$ and poles $\xi_{k}$. This implies that, for FPIMs, the described optimization process is required only once.

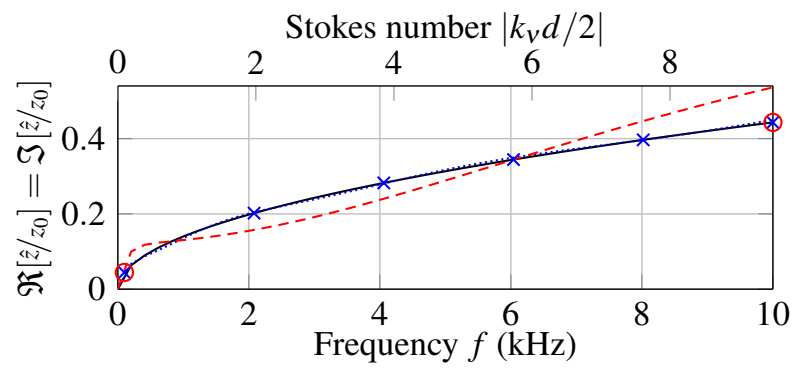

FIG. 2. (Color online) Broadband approximation of the Crandall model (7), for a perforation of length $l=0.8 \mathrm{~mm}$ and diameter $d=$ $0.3 \mathrm{~mm}$, typical of aeronautical micro-perforated liners. Real poles $\xi_{k}$ are linearly placed between $10 \mathrm{~Hz}$ and $10 \mathrm{kHz}$. (-): fractional term

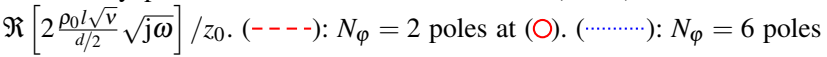
at $(\times)$. (Optimization with $w(\omega)=\omega$.)

For numerical simulations, it is of paramount importance that the TDIBC does not adversely impact numerical 
stability (i.e. that it is neutral on the CFL condition, if applicable). In that respect, the key parameter of (60) is the value of the greatest pole, denoted $\xi_{\max }$ : the higher $\xi_{\max }$, the lower the admissible time-step (remember that $N_{\xi}$ ODEs (50) now have to be integrated alongside the acoustic variables in the domain). Therefore, the ability to choose the poles (or, at least, $\left.\xi_{\max }\right)$ is beneficial. For instance, in Ref. $38, \xi_{\max }$ is chosen to be inferior to the maximum resolved angular frequency of the spatial discretization scheme.

In this section, the diffusive representation (40) of the convolution kernel $h_{1}^{\alpha}$ enabled to recast FPIMs (29) using only first-order ODEs (50): see (48) and (58). FPIMs can therefore be described as consisting of a constant resistance $a_{0}$, a mass reactance $a_{1} s$ and two diffusive parts: they are a particular case of partly diffusive impedance models, which are covered in the next section.

\section{PARTLY DIFFUSIVE IMPEDANCE MODELS IN THE TIME DOMAIN}

The purpose of this section is to further the study of Sec. IV, by designing TDIBCs for models other than FPIMs, which are mostly low or high-frequency approximations. More specifically, the models covered herein are those that are partly diffusive, referred to as partly diffusive impedance models (PDIMs). (In particular, FPIMs are PDIMs.) As a rule, physically, PDIMs are impedance models applicable to perforations and semi-infinite ground layers. This section presents the analysis of a perforation model in Sec. A, and of three semi-infinite ground layer models in Sec. B. It ends with a discussion of multi-pole models within the framework of PDIMs.

\section{A. Atalla and Sgard perforation model}

The Atalla and Sgard perforation model (12) can be written without approximations as

$$
(\Re[s]>0) \quad \hat{z}_{2}(s)=a_{\frac{1}{2}} \sqrt{1+\tau s}+a_{1} s,
$$

where the coefficients $a$. and the time-constant $\tau$ are nonnegative. To convert (63) in the time domain using a diffusive representation, its diffusive part $\hat{h}_{2}$ must be identified. Intuitively, $\hat{h}_{2}$ must be (at least) multivalued and decreasing at infinity, so that the methodology laid out in Sec. IV can be applied. Heuristically, the diffusive part is found to be

$$
\hat{h}_{2}(s):=\frac{\sqrt{1+\tau s}-1}{s} .
$$

It is a multivalued function, who can be extended to the left half-plane with a cut $\mathscr{C}_{2}$ in $]-\infty,-\tau^{-1}$ [. It admits a diffusive representation (43) whose diffusive weight $\mu_{2}$ can be computed from the jump across the cut (41), which yields

$$
\mu_{2}(\xi)=\frac{1}{\pi} \frac{\sqrt{\tau \xi-1}}{\xi} \mathbf{1}_{\mathscr{C}_{2}}(\xi) \quad\left(\mathscr{C}_{2}:=\right] \tau^{-1},+\infty[) .
$$

Compared to the diffusive weight $\mu_{1}$ of FPIMs (42), here, $\mu_{2}$ is null for any angular frequency $\xi$ below $\tau^{-1}$ : this indicates that the dynamic of $\hat{h}_{2}$ is comprised of a continuum of first-order systems whose time constants are strictly superior to $\tau$.

This analysis enables to recast the Atalla and Sgard model (63) as

$$
\begin{aligned}
\hat{z}_{2}(s) & =a_{\frac{1}{2}}+a_{\frac{1}{2}} \hat{h}_{2}(s) s+a_{1} s \\
& =a_{\frac{1}{2}}+a_{\frac{1}{2}} \int_{\tau^{-1}}^{\infty} \mu_{2}(\xi) \frac{s}{s+\xi} \mathrm{d} \xi+a_{1} s .
\end{aligned}
$$

The identity (67) demonstrates that the Atalla and Sgard model is a PDIM, which consists in a pure resistance $a_{\frac{1}{2}}$, a mass reactance $a_{1} s$, and a diffusive part $\hat{h}_{2}(s)$. In the time domain, it translates into the following causal integrodifferential equation:

$$
p=a_{\frac{1}{2}} u_{n}+a_{\frac{1}{2}} \int_{\tau^{-1}}^{\infty} \mu_{2}(\xi)\left(e^{-\xi} \star \dot{u}_{n}\right) \mathrm{d} \xi+a_{1} \dot{u}_{n} .
$$

Using the identity (57), the continuous TDIBC eventually reads

$$
p=a_{\frac{1}{2}} u_{n}+a_{\frac{1}{2}} \int_{\tau^{-1}}^{\infty} \mu_{2}(\xi)\left[-\xi \varphi_{\xi}+u_{n}\right] \mathrm{d} \xi+a_{1} \dot{u}_{n},
$$

where the diffusive variable $\varphi_{\xi}$ obey the ODE (50) with the non-null initial condition $\varphi_{0}(\xi)=u_{n}(0) / \xi$. The discretization of (69), as well as the broadband optimization of the $N_{\varphi}$ gains $\tilde{\mu}_{k}$ and poles $\xi_{k}$ can be done through the exact same process as that presented in Sec. IV. The only notable difference is that the poles $\xi_{k}$ now obey $\xi_{k}>\tau^{-1}$. The present analysis has shown how the Atalla and Sgard model should be numerically approximated.

\section{B. "Square-root type" semi-infinite ground layer model}

This section is dedicated to the analysis of "square-root type" characteristic impedance models (18) used for semiinfinite ground layers (5). Following the table I of Ref. 28, three cases must be distinguished, each one covering a different set of models, depending on the values of the angular frequencies $\omega_{i}$. They are all PDIMs, and, as such, their analysis follows the same methodology as that exposed for the Atalla and Sgard model. Therefore, developments have been kept concise.

The first case consists in $\omega_{2}$ and $\omega_{3}$ being null; the model then reduces to

$$
(\Re[s]>0) \quad \hat{z}_{31}(s)=q_{z} \frac{\sqrt{s+\omega_{1}}}{\sqrt{s}} .
$$

It is a PDIM, whose diffusive part $\hat{h}_{31}$ can be extended to the left half-plane by defining a cut $\mathscr{C}_{31}$ in $]-\omega_{1}, 0[$; it can be recast as

$$
\begin{aligned}
\hat{z}_{31}(s) & =q_{z}+q_{z} \hat{h}_{31}(s) \\
& =q_{z}+q_{z} \int_{0}^{\omega_{1}} \frac{\mu_{31}(\xi)}{s+\xi} \mathrm{d} \xi,
\end{aligned}
$$


where the diffusive weight $\mu_{31}$ is computed from (41):

$$
\mu_{31}(\xi)=\frac{1}{\pi} \frac{\sqrt{\omega_{1}-\xi}}{\sqrt{\xi}} \mathbf{1}_{\mathscr{C}_{31}}(\xi) \quad\left(\mathscr{C}_{31}:=\right] 0, \omega_{1}[) .
$$

Here, the diffusive weight is null for $\xi>\omega_{1}$ : the dynamic of $\hat{h}_{31}$ is comprised of a continuum of first-order systems whose time constants are strictly inferior to $1 / \omega_{1}$. The corresponding continuous TDIBC is:

$$
p=q_{z} u_{n}+q_{z} \int_{0}^{\omega_{1}} \mu_{31}(\xi) \varphi_{\xi} \mathrm{d} \xi
$$

where the diffusive variables $\varphi_{\xi}$ obey (50) with the null initial condition $\varphi_{0}(\xi)=0$.

The second case consists in $\omega_{1}=\omega_{2}$, which leads to the following model:

$$
(\Re[s]>0) \quad \hat{z}_{32}(s)=q_{z} \frac{s+\omega_{1}}{\sqrt{s\left(s+\omega_{3}\right)}} .
$$

It is a PDIM, which can be written as

$$
\begin{aligned}
\hat{z}_{32}(s) & =q_{z} \hat{h}_{32}(s)\left(s+\omega_{1}\right) \\
& =q_{z} \int_{0}^{\omega_{3}} \mu_{32}(\xi) \frac{s+\omega_{1}}{s+\xi} \mathrm{d} \xi
\end{aligned}
$$

where the diffusive weight $\mu_{32}$ of the diffusive part $\hat{h}_{32}$ is, the cut being on $]-\omega_{3}, 0[$ :

$$
\mu_{32}(\xi)=\frac{1}{\pi} \frac{1}{\sqrt{\xi\left(\omega_{3}-\xi\right)}} \mathbf{1}_{\mathscr{C}_{32}}(\xi) \quad\left(\mathscr{C}_{32}:=\right] 0, \omega_{3}[) .
$$

The corresponding time-domain equation is slightly different from the ones covered so far:

$$
p=q_{z} \int_{0}^{\omega_{3}} \mu_{32}(\xi)\left(e^{-\xi} \star\left(\dot{u}_{n}+\omega_{1} u_{n}\right)\right) \mathrm{d} \xi .
$$

Using the diffusive variables (49), the continuous TDIBC reads

$$
p=q_{z} \int_{0}^{\omega_{3}} \mu_{32}(\xi)\left[-\left(\xi-\omega_{1}\right) \varphi_{\xi}+u_{n}\right] \mathrm{d} \xi,
$$

where the diffusive variables obey (50) with the non-null initial condition $\varphi_{0}(\xi)=\frac{u_{n}(0)}{\xi-\omega_{1}}$.

The third and last case consists in $\omega_{1}, \omega_{2}$, and $\omega_{3}$ both non-null and different from one another:

$$
(\Re[s]>0) \quad \hat{z}_{33}(s)=q_{z} \sqrt{\frac{\left(s+\omega_{1}\right)\left(s+\omega_{2}\right)}{s\left(s+\omega_{3}\right)}} .
$$

It is also a PDIM, which can be recast as

$$
\begin{aligned}
\hat{z}_{33}(s) & =q_{z}+q_{z} \hat{h}_{33}(s) \\
& =q_{z}+q_{z} \int_{0}^{\infty} \frac{\mu_{33}(\xi)}{s+\xi} \mathrm{d} \xi .
\end{aligned}
$$

The corresponding continuous TDIBC is therefore

$$
p=q_{z} u_{n}+q_{z} \int_{0}^{\infty} \mu_{33}(\xi) \varphi_{\xi} \mathrm{d} \xi
$$

where the diffusive variables $\varphi_{\xi}$ obey (50) with $\varphi_{0}(\xi)=0$. However, the complication of this case lies in the fact that the cut depends upon the position of $\omega_{3}$ relative to $\omega_{1}$. Using the expression (41), we can write

$$
\mu_{33}(\xi)=\frac{1}{\pi} \sqrt{\left|\frac{\left(\xi-\omega_{1}\right)\left(\xi-\omega_{2}\right)}{\xi\left(\xi-\omega_{3}\right)}\right|} l(\xi),
$$

where the function $l(\xi)$ reflects the location of the cut on the negative real axis. For $\omega_{3}>\omega_{2}$ (beware of the minus sign), $\imath(\xi)=\mathbf{1}_{] 0, \omega_{1}[}(\xi)-\mathbf{1}_{] \omega_{2}, \omega_{3}[}(\xi)$, for $\omega_{3}$ between $\omega_{1}$ and $\omega_{2}, l(\xi)=\mathbf{1}_{] 0, \omega_{1}[}(\xi)+\mathbf{1}_{] \omega_{3}, \omega_{2}[}(\xi)$, and for $\omega_{3}<\omega_{1}$, $\imath(\xi)=\mathbf{1}_{] 0, \omega_{3}[}(\xi)+\mathbf{1}_{] \omega_{1}, \omega_{2}[}(\xi)$.

\section{Link with multi-pole models}

In Sec. IV and $\mathrm{V}$, six partly diffusive models $(45,55,67,72,77,82)$ have been covered. They can be written in a compact manner as

$$
\hat{z}(s)=a_{0}+a_{\mu} \hat{h}_{\mu}(s)+a_{\eta} \hat{h}_{\eta}(s) s+a_{1} s,
$$

where the coefficients $a$. are positive, and $\hat{h}_{\bullet}$ denotes the diffusive representation (43) with the diffusive weight $\bullet$. For three of these models, namely $(45,72,82)$, the coefficient $a_{\eta}$ is null; once discretized, they reduce to

$$
\hat{z}(s)=a_{0}+a_{\mu} \sum_{k=1}^{N_{\varphi}} \frac{\tilde{\mu}_{k}}{s+\xi_{k}}+a_{1} s
$$

where the gains $\tilde{\mu}_{k}$ are real. The expression (86) is that of a multi-pole model, as found in the aeroacoustics literature, ${ }^{15,17}$ with three notable specificities. Firstly, the coefficients $a$. are modeled, not optimized. The gains and poles follow from the optimization process presented in Sec. D. Note that, for the models $(45,55,67,72,77)$, the diffusive part $\hat{h}_{\bullet}$ is independent of the material (or can be made independent using a linear change of variable). The optimization process is then required only once. Secondly, the poles $\xi_{k}$ may be restricted to a given band, as is the case for $(72,82)$. Lastly, the poles $\xi_{k}$ are real. Although it is possible for a discrete diffusive representation to lead to complex poles, ${ }^{39}$ this is not the case for the acoustical models studied so far. A family of models which exhibits complex poles is covered in the next section. 


\section{PARTLY OSCILLATORY-DIFFUSIVE IMPEDANCE MODELS IN THE TIME DOMAIN}

The purpose of this section is to study backing cavity impedance models (4), thus completing the analysis of the models given in Sec. III. The model analyzed herein is (4) with a fractional polynomial wavenumber:

$$
(\Re[s]>0) \quad \hat{z}_{4}(s)=z_{c} \operatorname{coth}\left[\left(a_{0}+a_{\alpha} s^{\alpha}+a_{1} s\right) l_{c}\right],
$$

where $\alpha \in] 0,1[$, the coefficients $a$. are non-negative, and $z_{c}$ is frequency-independent. This model is simple enough to permit a semi-analytical derivation of its time-domain representation, yet rich enough to cover a wide range of acoustical models: $(17,28)$ and approximations of $(21,25)$. Moreover, without the fractional term, i.e. for $a_{\alpha}=0$, (87) reduces to the EHR numerical model, ${ }^{20}$ widely used for numerical simulations.

This section is organized as follows. Sec. A presents the derivation of the discrete TDIBC. Sec. B illustrates the achievable broadband approximations.

\section{A. Time-domain representation}

The first step to derive the time-domain representation is to recast (4) as

$$
\hat{z}_{4}(s)=z_{c}+2 z_{c} \frac{e^{-2 \mathrm{j} k_{c}(s) l_{c}}}{1-e^{-2 \mathrm{j} k_{c}(s) l_{c}}},
$$

which can be rewritten, using the expression of $\mathrm{j} k_{c}$ :

$$
\hat{z}_{4}(s)=z_{c}+2 z_{c} \Upsilon e^{-\Delta t s} \hat{h}_{4}(s),
$$

with the dimensionless coefficient $\Upsilon:=e^{-2 a_{0} l_{c}}<1$, the time delay $\Delta t:=2 a_{1} l_{c}$, and the function

$$
\hat{h}_{4}(s):=\frac{e^{-2 a_{\alpha} l_{c} s^{\alpha}}}{1-\Upsilon e^{-2\left(a_{1} s+a_{\alpha} s^{\alpha}\right) l_{c}}} .
$$

At first sight, the identity (89) may seem analogous to those derived for PDIMs, such as (66) or (71). However, the first major difference is the exponential factor, which translates in the time domain into a delay:

$$
p(t)=z_{c} u_{n}(t)+2 z_{c} \Upsilon\left[h_{4}(\cdot-\Delta t) \star u_{n}\right](t) .
$$

Physically, $\Delta t$ is the time it takes a normal incidence plane wave to travel in and out of the inviscid cavity: $\Delta t=2 l_{c} / v_{g}$, where $v_{g}$ denotes the group velocity. The second major difference lies in the nature of $\hat{h}_{4}$, which does not admit a diffusive representation (43). Indeed, although $\hat{h}_{4}$ is a multivalued function which can be extended to the left half-plane with a cut in $\mathbb{R}_{-}$, it also has poles. ${ }^{40}$ Using the residue theorem and Jordan's lemma (as in (39)) leads to the following representation

$$
\begin{aligned}
& h_{4}(t)=r_{0} e^{s_{0} t}+2 \sum_{n=1}^{\infty} \Re\left[r_{n} e^{s_{n} t}\right]+\int_{0}^{\infty} \mu_{4}^{\alpha}(\xi) e^{-\xi t} \mathrm{~d} \xi \\
& \hat{h}_{4}(s)=\frac{r_{0}}{s-s_{0}}+\sum_{n=1}^{\infty} \frac{r_{n}}{s-s_{n}}+\frac{r_{n}^{*}}{s-s_{n}^{*}}+\int_{0}^{\infty} \frac{\mu_{4}^{\alpha}(\xi)}{s+\xi} \mathrm{d} \xi
\end{aligned}
$$

which is split between an oscillatory part, associated with the poles $s_{n}$ and their complex residues $r_{n}$, and a diffusive part, linked to the fractional term $s^{\alpha}$ through the diffusive weight $\mu_{4}^{\alpha}$. The representation (92) is called the oscillatorydiffusive (also poles and cuts) ${ }^{24}$ representation of $h_{4}$. The impedance model $\hat{z}_{4}$ is said to be a partly oscillatorydiffusive impedance model (PODIM), whose oscillatorydiffusive part is $\hat{h}_{4}$. As illustrated in Sec. B, the poles $s_{n}$ are associated to anti-resonances in the frequency domain; they solve the following fractional polynomial equation in $\mathbb{C}$ (which may not admit a solution for a given $n$ )

$$
(n \in \mathbb{Z}) \quad a_{1} s_{n}+a_{\alpha} s_{n}^{\alpha}=-a_{0}+\mathrm{j} \frac{n \pi}{l_{c}},
$$

and the complex residues $r_{n}$ are given by

$$
r_{n}:=\operatorname{Res}\left(\hat{h}_{4}, s_{n}\right)=\frac{e^{-2 a_{\alpha} l_{c} s_{n}^{\alpha}}}{2\left(a_{1}+\alpha a_{\alpha} s_{n}^{\alpha-1}\right) l_{c}} .
$$

The poles are paired (i.e. $s_{n}$ is the complex conjugate of $\left.s_{-n}\right)$, which reflects the reality condition. The stability condition implies that each pole has a negative real part. Lastly, the diffusive weight, which is subject to the well-posedness condition (44), is given by, for $\xi>0$,

$$
\mu_{4}^{\alpha}(\xi)=\frac{1}{\pi} \frac{\sin \left(2 a_{\alpha} l_{c} \xi^{\alpha}\right)}{1-2 \Upsilon e^{\Delta t \xi} \cos \left(2 a_{\alpha} l_{c} \xi^{\alpha}\right)+\Upsilon^{2} e^{2 \Delta t \xi}} .
$$

Without a fractional term (i.e. $a_{\alpha}=0$ ), the diffusive weight is null, and the representation (92) is purely oscillatory.

By injecting (92) into (91), one can express the continuous TDIBC associated with $\hat{z}_{4}$ as

$$
\begin{aligned}
& p(t)=z_{c} u_{n}(t)+2 z_{c} \Upsilon\left[r_{0} \psi_{0}(t-\Delta t)\right. \\
& \left.+2 \sum_{n=1}^{\infty} \Re\left[r_{n} \psi_{n}\right](t-\Delta t)+\int_{0}^{\infty} \mu_{4}^{\alpha}(\xi) \varphi_{\xi}(t-\Delta t) \mathrm{d} \xi\right]
\end{aligned}
$$

where there are now two families of additional variables: the diffusive variables $\varphi_{\xi}$, defined by (49), which obey the first-order real-valued ODE (50) with null initial condition $\varphi_{0}(\xi)=0$, and the oscillatory variables $\psi_{n}:=e^{s_{n} \cdot} \star u_{n}$, which obey the following first-order complex-valued ODE:

$$
\left(n \in \mathbb{N}^{*}\right) \quad \dot{\psi}_{n}(t)=s_{n} \psi_{n}(t)+u_{n}(t) \quad \psi_{n}(0)=0 .
$$

In contrast with the TDIBCs derived in Secs. IV and V $(48,58,69,74,80,83)$, the TDIBC (97) is expressed through delayed first-order ODEs.

From the above analysis, the following discrete TDIBC can be proposed

$$
\hat{z}_{4}^{\text {num }}(s)=1+2 \Upsilon e^{-\Delta t} \hat{h}_{4}^{\text {num }}(s),
$$


where the oscillatory-diffusive part $\hat{h}_{4}$ is approximated by

$$
\hat{h}_{4}^{\text {num }}(s):=\frac{\tilde{r}_{0}}{s-s_{0}}+\sum_{n=1}^{N_{\psi}-1} \frac{\tilde{r}_{n}}{s-s_{n}}+\frac{\tilde{r}_{n}^{*}}{s-s_{n}^{*}}+\sum_{k=1}^{N_{\varphi}} \frac{\tilde{\mu}_{k}}{s+\xi_{k}},
$$

whose DoF are the $N_{\psi}$ complex poles $s_{n}$ and gains $\tilde{r}_{n}$, and the $N_{\varphi}$ real poles $\xi_{k}$ and gains $\tilde{\mu}_{k}$. To tune $\hat{h}_{4}^{\text {num }}$, an optimization procedure similar to that presented in Sec. IV can be followed: the poles $\xi_{k}$ are chosen, the poles $s_{n}$ are $o b$ tained from solving (94), and the gains $\tilde{\mu}_{k}, \tilde{r}_{n}$ are computed from the minimization of a least-square distance between $\hat{h}_{4}$ and $\hat{h}_{4}^{\text {num }}$, which reduces to computing a pseudo-inverse. Note that the expression of $\hat{h}_{4}$ given by (90) depends upon $a_{0}, a_{\alpha}$ and $a_{1}$. Any change of variables will leave at least two dependencies. Therefore, in contrast to Sec. V, the optimization process does depend upon the material.

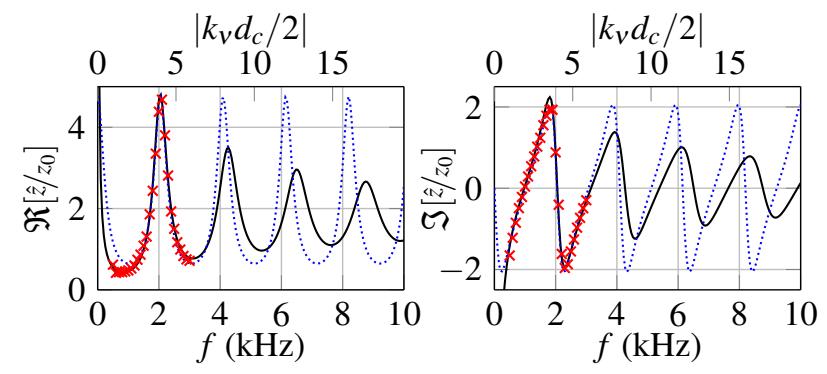

FIG. 3. (Color online) CT57 Broadband modeling. (x): educed impedance. $^{41}$ (—): fractional model (101). (……...): EHR model (102). (The cavity cut-off frequency is above $300 \mathrm{kHz}$.)

\section{B. Application on a ceramic tubular liner}

To illustrate the capability of the numerical model (99) to realize both a parsimonious and broadband approximation of a physical model, it is herein applied to two models. A fractional one

$$
\hat{z}_{\mathrm{Fr}}(s)=\frac{z_{c}}{\sigma} \operatorname{coth}\left[\left(a_{\frac{1}{2}} \sqrt{s}+a_{1} s\right) l_{c}\right],
$$

and the EHR model (without its polynomial part)

$$
\hat{z}_{\mathrm{EHR}}(s)=\frac{z_{c}}{\sigma} \operatorname{coth}\left[\left(a_{0}+a_{1} s\right) l_{c}\right]
$$

Using, for instance, the Bruneau wavenumber (25), both models can be fit to experimental data without optimization. A widely-used benchmark is the liner CT57. ${ }^{41}$ It is a ceramic tubular liner, whose dimensions are a cavity length $l_{c}=85.6 \mathrm{~mm}$, a cavity diameter $d_{c}=0.6 \mathrm{~mm}$, and a porosity $\sigma=57 \%$. In Fig. 3 is proposed a comparison between experimental data, the fractional model (101) and the EHR model (102). Both models accurately match the experimental data, but differ at both low frequencies (see the EHR's reactance) and high frequencies. These differences stem from the two different modelings of visco-thermal losses: through a fractional term $a_{\frac{1}{2}} \sqrt{s}$ or a frequency-independent constant $a_{0}$. The former, which is physically-based, is expected to be more representative than the latter. However, additional experimental data (especially over a broader frequency range) could help favor one over the other.

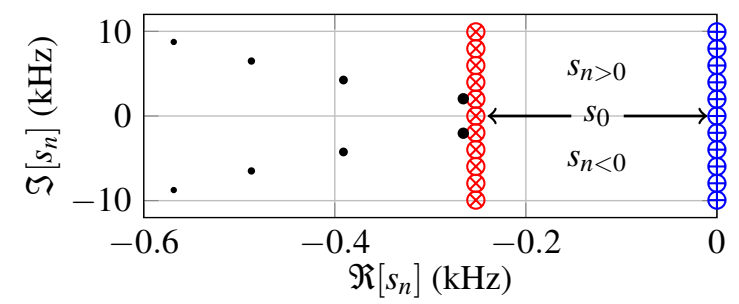

FIG. 4. (Color online) First poles $\left(s_{n}\right)_{n}$ of $\hat{h}_{4}$, given by (94), for three models. (๑): fractional (101). (®): EHR (102). ( () : inviscid, i.e. (102) with $a_{0}=0$. Marker area is proportional to residue magnitude $\left|r_{n}\right|$ (95).

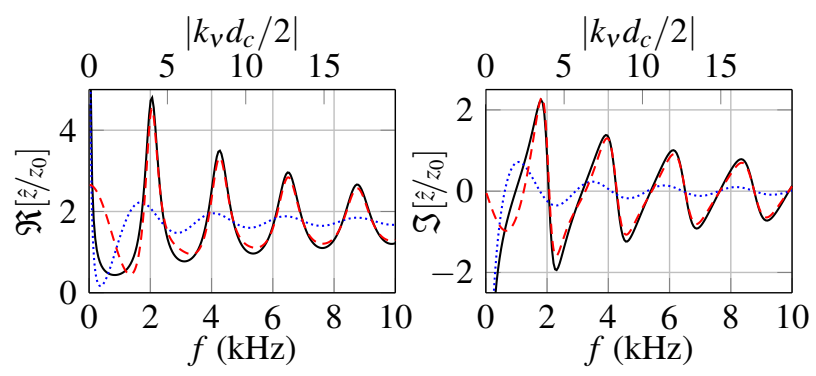

FIG. 5. (Color online) Analysis of the fractional model (101) using (89). (-): full model (101). (----): diffusive part of $\hat{h}_{4}$ neglected (i.e. oscillatory part $\left(r_{n}\right)_{n},\left(s_{n}\right)_{n}$ only). ( $\left.\cdots \cdots \cdots \cdots . . ..\right)$ : oscillatory part of $\hat{h}_{4}$ neglected (i.e. diffusive part $\mu_{4}^{\alpha}$ only).

To build an approximation to (101) or (102) using the discrete TDIBC (99), a balance has to be struck between an accurate approximation of the oscillatory part $\left(N_{\psi}\right)$ and of the diffusive part $\left(N_{\varphi}\right)$. To that end, insights can be gained from comparing their relative importance. A plot of the first pairs of complex poles is proposed in Fig. 4, where the marker size reflects the magnitude of the associated residue. For the fractional model, the sequence of residues convergences toward zero, while it remains constant for the EHR model (see (95)). Practically, this implies that $N_{\psi}$ can be chosen smaller for the fractional model.

As the EHR model does not have a fractional term, it only has an oscillatory part; therefore, its numerical approximation verifies $N_{\varphi}=0$. By contrast, Fig. 5 shows that both the oscillatory and diffusive parts contribute to the fractional model, each in a specific manner. The diffusive part is important at low frequency, while the oscillatory part models the successive anti-resonances: the $n$-th pair of poles enables to capture the $n$-th anti-resonance.

Broadband approximations of (101) and (102), using the optimization process presented earlier, are presented in 
Fig. 6 and 7. It shows that the present analysis can help build broadband approximations with only an elementary linear least squares optimization.

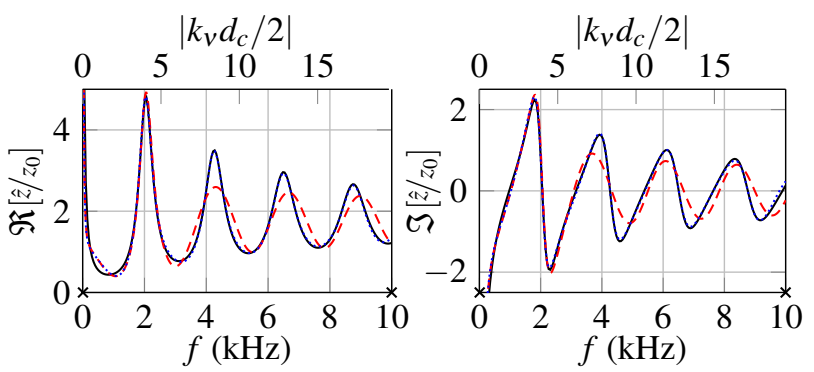

FIG. 6. (Color online) Broadband approximation of the fractional model (101) with (99). ( - ): model (101). (----): first pair of complex poles $\left(N_{\psi}=2\right)$ ( $\rightarrow$ first anti-resonance). ( $\left.\cdots \cdots \cdots \cdots \cdot \cdots\right): 4$ pairs of complex poles $\left(N_{\psi}=\right.$ 8) ( $\rightarrow 4$ anti-resonances). (x): $N_{\varphi}=2$ poles $\xi_{k}$ at $10 \mathrm{~Hz}$ and $10 \mathrm{kHz}$ for the diffusive part. (Optimization with $w(\omega)=1$.)

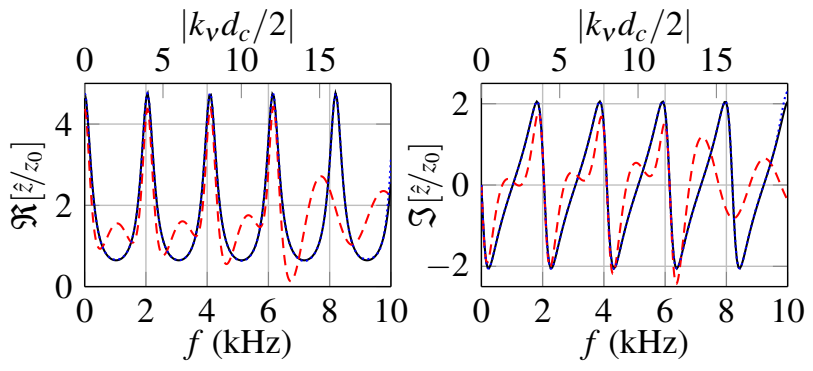

FIG. 7. (Color online) Broadband approximation of the EHR model (102) with (99). (- - ): model (102). (--- ) : 3 pairs of complex poles $\left(N_{\psi}=\right.$ 7). (…….): 7 pairs of complex poles $\left(N_{\psi}=15\right)$. No diffusive part $\left(N_{\varphi}=0\right)$. (Optimization with $w(\omega)=1$.)
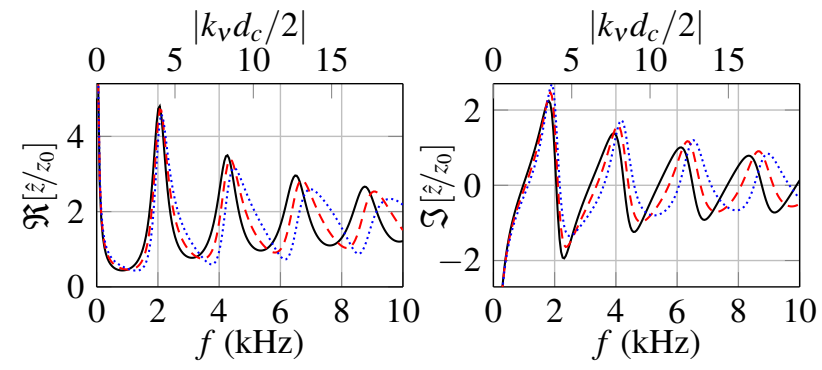

FIG. 8. (Color online) Impact of approximating the delay $\Delta t$ in (89). ($10 \%$ error on the delay.

In numerical simulations, to benefit from the broadband capacity of (99), the delay $\Delta t$ must be approximated as accurately as possible, as illustrated in Fig. 8, which shows that an error of a few percent is enough to cause significant differences at high frequency. However, this is numerically costly, as it requires additional memory.

The link between (physical) cavity impedance models and (numerical) multi-pole models is provided by $(89,93)$. With a neglected delay, (99) formally reduces to a multipole model, with both real and complex poles. The real poles $\xi_{k}$ are associated with the diffusive part of (93) (viscous diffusion in the cavity modeled with the $s^{\alpha}$ term), while the complex poles $s_{n}$ are associated with its oscillatory part. Numerically, the former are chosen (following the optimization strategy presented in Sec. D), while the latter are computed from solving (94).

\section{CONCLUSIONS}

This paper has shown an analysis of acoustical models, which relies on the oscillatory-diffusive representation, that enables to derive theoretically exact continuous TDIBCs. The corresponding discrete TDIBCs have been shown able to yield broadband approximations using the output of a mere linear least squares optimization that is, for most models, required only once, as it is independent of the material geometry. Models drawn from both duct acoustics and outdoor sound propagation have been covered. Overall, each model has lead to a different TDIBC; this contrasts with a purely empirical one-size-fits-all approach.

Models for both perforation and semi-infinite ground layers have been covered in Sec. IV and V. Structurally, they have been shown to be partly diffusive impedance models (PDIMs), and lead to time-local TDIBCs $(48,58,69,74,80,83)$ that consist of first-order ODEs. Some of them have been singled out as multi-pole models $(48,74,83)$, widely employed in the literature. Additionally, fractional calculus concepts have been used to show the admissibility of fractional polynomial impedance models (FPIMs), see (35).

Sec. VI has covered a cavity impedance model with a fractional polynomial wavenumber. It has been shown to exhibit an oscillatory and a diffusive part, both of which are delayed, due to the back-and-forth wave motion within the cavity. The derived TDIBC (97) has been recast using delayed first-order ODEs, which provides, for instance, an alternative implementation of the EHR model.

We now list suggestions to further this work. Firstly, the presented analysis could be extended to cover acoustical models which involve Bessel or hyperbolic functions $(6,9,25)$, as these have only been covered under an approximated form herein. Secondly, the benefit in using a nonlinear least squares optimization method to optimize simultaneously the gains $(\tilde{\mu}, \tilde{r})$ and (real) poles $\xi$ could be investigated. Lastly, a study of models that cover nonlinear effects, of particular interest when dealing with perforated plates, would be a significant advancement to the timedomain simulation of sound absorption. 


\section{ACKNOWLEDGMENTS}

This research has been financially supported by the French ministry of defense (Direction Générale de l'Armement) and ONERA (the French Aerospace Lab). The authors are grateful to Dr. Michael Jones (NASA) for providing the CT57 experimental data.

\section{REFERENCES}

${ }^{1}$ C. Tam, Computational aeroacoustics: A wave number approach (Cambridge University Press, Cambridge, 2012), Chap. 10, pp. 181-182.

${ }^{2}$ R. Astley, R. Sugimoto, and P. Mustafi, "Computational aero-acoustics for fan duct propagation and radiation. current status and application to turbofan liner optimisation," J. Sound Vib. 330(16), 3832-3845 (2011).

${ }^{3}$ I. Crandall, Theory of vibrating systems and sound (D. Van Nostrand Company, New York, 1926), App. A.

${ }^{4}$ M. Bruneau, Fundamentals of acoustics (ISTE, London, 2013), 637 p.

${ }^{5} \mathrm{~T}$. Melling, "The acoustic impedance of perforates at medium and high sound pressure levels," J. Sound Vib. 29(1), 1-65 (1973).

${ }^{6}$ Y. Miki, "Acoustical properties of porous materials - Modifications of Delany-Bazley models,” J. Acoust. Soc. Jpn. (E) 11(1), 19-24 (1990).

${ }^{7}$ C. Richter, J. Hay, L. Panek, N. Schönwald, S. Busse, and F. Thiele, "A review of time-domain impedance modelling and applications," J. Sound Vib. 330(16), 3859-3873 (2011).

${ }^{8}$ B. Cotté and P. Blanc-Benon, "Time-domain simulations of sound propagation in a stratified atmosphere over an impedance ground,' J. Acoust. Soc. Am. 125(5), EL202-EL207 (2009).

${ }^{9}$ D. Botteldooren, "Finite-difference time-domain simulation of lowfrequency room acoustic problems," J. Acoust. Soc. Am. 98(6), 33023308 (1995).

${ }^{10} \mathrm{~S}$. Davis, "Low-dispersion finite difference methods for acoustic waves in a pipe," J. Acoust. Soc. Am. 90(5), 2775-2781 (1991).

${ }^{11} \mathrm{C}$. Tam and L. Auriault, "Time-domain impedance boundary conditions for computational aeroacoustics," AIAA J. 34(5), 917-923 (1996).

${ }^{12}$ Y. Özyörük, L. N. Long, and M. G. Jones, "Time-domain numerical simulation of a flow-impedance tube," J. Comput. Physics 146(1), 29-57 (1998).

${ }^{13}$ R. Luebbers, F. P. Hunsberger, K. S. Kunz, R. B. Standler, and M. Schneider, "A frequency-dependent finite-difference time-domain formulation for dispersive materials," IEEE Trans. Electromagn. Compat. 32(3), 222227 (1990).

${ }^{14}$ K.-Y. Fung and H. Ju, "Broadband time-domain impedance models," AIAA J. 39(8), 1449-1454 (2001).

${ }^{15}$ Y. Reymen, M. Baelmans, and W. Desmet, "Time-domain impedance formulation based on recursive convolution," AIAA Paper 2006-2685.

${ }^{16}$ V. Ostashev, S. Collier, D. Wilson, D. Aldridge, N. Symons, and D. Marlin, "Padé approximation in time-domain boundary conditions of porous surfaces," J. Acoust. Soc. Am. 122(1), 107-112 (2007).

${ }^{17}$ X. Y. Li, X. D. Li, and C. Tam, "Improved multipole broadband time-domain impedance boundary condition," AIAA J. 50(4), 980-984 (2012).

${ }^{18}$ J. Bin, M. Hussaini, and S. Lee, "Broadband impedance boundary conditions for the simulation of sound propagation in the time domain," $\mathrm{J}$. Acoust. Soc. Am. 125(2), 664-675 (2009).

${ }^{19}$ D. Dragna, P. Pineau, and P. Blanc-Benon, "A generalized recursive convolution method for time-domain propagation in porous media," J. Acoust. Soc. Am. 138(2), 1030-1042 (2015).

${ }^{20} \mathrm{~S}$. Rienstra, "Impedance models in time domain, including the extended Helmholtz resonator model,” AIAA Paper 2006-2686.
${ }^{21}$ N. Chevaugeon, J.-F. Remacle, and X. Gallez, "Discontinuous Galerkin implementation of the extended Helmholtz resonator model in time domain," AIAA Paper 2006-2569.

${ }^{22}$ L. Pascal, E. Piot, and G. Casalis, "A new implementation of the extended Helmholtz resonator acoustic liner impedance model in time domain CAA," J. Comput. Acoust. 24(1), 1550015 (2016).

${ }^{23}$ L. Zhou and H. Bodén, "A systematic uncertainty analysis for liner impedance eduction technology," J. Sound Vib. 356, 86 - 99 (2015).

${ }^{24} \mathrm{~T}$. Hélie and D. Matignon, "Diffusive representations for the analysis and simulation of flared acoustic pipes with visco-thermal losses," Math. Mod. Meth. App. S. 16(04), 503-536 (2006).

${ }^{25}$ R. Kirby and A. Cummings, "The impedance of perforated plates subjected to grazing gas flow and backed by porous media," J. Sound Vib. 217(4), 619-636 (1998).

${ }^{26}$ M. Myers, "On the acoustic boundary condition in the presence of flow," J. Sound Vib. 71(3), 429-434 (1980).

${ }^{27} \mathrm{Q}$. Zhang and D. J. Bodony, "Numerical investigation of a honeycomb liner grazed by laminar and turbulent boundary layers," J. of Fluid Mech. 792, 936-980 (2016).

${ }^{28}$ D. Dragna and P. Blanc-Benon, "Physically admissible impedance models for time-domain computations of outdoor sound propagation," Acta Acust. United Ac. 100(3), 401-410 (2014).

${ }^{29}$ A. Zemanian, Distribution theory and transform analysis (McGraw-Hill, New York, 1965), $371 \mathrm{p}$.

${ }^{30}$ D.-Y. Maa, "Potential of microperforated panel absorber," J. Acoust. Soc. Am. 104(5), 2861-2866 (1998).

${ }^{31}$ N. Atalla and F. Sgard, "Modeling of perforated plates and screens using rigid frame porous models," J. Sound Vib. 303(1), 195-208 (2007).

${ }^{32}$ J. Allard and N. Atalla, Propagation of Sound in Porous Media: Modelling Sound Absorbing Materials, 2nd ed. (John Wiley \& Sons, Chichester, 2009), $374 \mathrm{p}$

${ }^{33}$ D. Li, D. Chang, and B. Liu, "Enhancing the low frequency sound absorption of a perforated panel by parallel-arranged extended tubes," App. Acoust. 102, 126-132 (2016).

${ }^{34}$ D. Matignon, "An introduction to fractional calculus," in Scaling, Fractals and Wavelets (ISTE - Wiley, London, 2009), pp. 237-277.

${ }^{35}$ M. Caputo, "Vibrations of an infinite plate with a frequency independent Q,” J. Acoust. Soc. Am. 60(3), 634-639 (1976).

${ }^{36} \mathrm{D}$. Duffy, Transform methods for solving partial differential equations, 2nd ed. (CRC press, Boca Raton, 2004), 713 p.

${ }^{37}$ E. Blanc, G. Chiavassa, and B. Lombard, "A time-domain numerical modeling of two-dimensional wave propagation in porous media with frequency-dependent dynamic permeability," J. Acoust. Soc. Am. 134(6), 4610-4623 (2013).

${ }^{38}$ F. Monteghetti, D. Matignon, E. Piot, and L. Pascal, "Simulation temporelle d'un modèle d'impédance de liner en utilisant la représentation diffusive d'opérateurs (Time-domain simulation of an acoustic liner impedance model using the diffusive representation of operators)," in 13e Congrès Français d'Acoustique (Le Mans, France, Apr. 11-15 2016), No. 000130, pp. 2549-2555.

${ }^{39}$ R. Mignot, T. Hélie, and D. Matignon, "On the singularities of fractional differential systems, using a mathematical limiting process based on physical grounds," Phys. Scripta T136(014023), (2009).

${ }^{40}$ D. Matignon and B. d'Andréa Novel, "Spectral and time-domain consequences of an integro-differential perturbation of the wave PDE," in Third international conference on mathematical and numerical aspects of wave propagation phenomena (SIAM, 1995), pp. 769-771.

${ }^{41}$ M. Jones, W. Watson, and T. Parrott, "Benchmark data for evaluation of aeroacoustic propagation codes with grazing flow," AIAA Paper 20052853. 